\title{
DIFFERENTIAL-BOUNDARY OPERATORS
}

BY

\author{
ALLAN M. KRALL $\left({ }^{1}\right)\left({ }^{2}\right)$
}

\begin{abstract}
Differential-boundary systems occur naturally as adjoints for ordinary differential systems involving integral boundary conditions. In this paper such systems are generalized so that the adjoint system has the same form as the original. Interior boundary points are introduced and removed, and the integrals, used in the boundary conditions, are also removed. Selfadjoint systems are classified, and an eigenfunction expansion is derived. Finally, nonselfadjoint systems are discussed and again, an eigenfunction expansion is derived.
\end{abstract}

I. Introduction. Perhaps the simplest example of a differential-boundary operator was given by Phillips [21] in a discussion of maximal dissipative operators. If $h \in L^{2}(0,1)$, and $\|h\| \leqq 2$, Phillips defined the operator $L$ by letting $L y=y^{\prime}-y$ $+y(0) h$ on a domain $D=\left\{y ; y\right.$ is absolutely continuous, $y$ and $\left.y^{\prime} \in L^{2}(0,1), y(1)=0\right\}$. The operator and its domain represent a system which involves some procedure for returning energy with an interior density of $y(0) h$.

The earliest paper related to differential-boundary operators appears to be due to Hilb [11] in 1911. He discussed the operator

$$
L y=\frac{d}{d x}\left(p(x) \frac{d y}{d x}\right)+q(x) y,
$$

where $y$ is defined on $[0,1]$ and satisfies boundary conditions

$$
\int_{0}^{1} K(x) y(x) d x+\beta y(0)-\alpha y^{\prime}(0)=0, \quad h_{1} y^{\prime}(1)-h_{2} y(1)=0 .
$$

Hilb defined an adjoint operator by $\left({ }^{3}\right)$

$$
L^{+} z=\frac{d}{d x}\left(\bar{p}(x) \frac{d z}{d x}\right)+\bar{q}(x) z-\bar{K}(x) z(0) / \bar{\alpha},
$$

where $z$ satisfies boundary conditions

$$
\bar{\beta} z(0)-\bar{\alpha} z^{\prime}(0)=0, \quad \bar{h}_{1} z^{\prime}(1)-\bar{h}_{2} z(1)=0 .
$$

Received by the editors November 3, 1969 and, in revised form, May 14, 1970.

AMS 1969 subject classifications. Primary 3420, 3430; Secondary 3436.

Key words and phrases. Operator, differential operator, boundary value problem, differential system, selfadjoint, nonselfadjoint, Sturm-Liouville problem, adjoint, eigenvalue, spectrum, spectral resolution.

(1) McAllister Building, The Pennsylvania State University, University Park, Pennsylvania 16802.

(2) This research was supported in part by NASA Grant NGR 39-009-041.

$\left({ }^{3}\right)$ This is the author's notation, not Hilb's.

Copyright (C) 1971, American Mathematical Society 
He succeeded in expanding functions in the domain of $L$ in a series of eigenfunctions of $L$ and in expanding functions in the domain of $L^{+}$in a series of eigenfunctions of $L^{+}$. The importance of this work is that the presence of the integral in the first boundary condition induces the boundary term in the adjoint operator.

Hilb's work was subsequently extended to the interval $[0, \infty)$ by his student Betschler [1], was further improved by the author [14], [15], and again extended by Kim [13].

An important application of differential-boundary operators may be found in the work of Feller [9], published in 1952. Feller was interested in a generalization of the Fokker-Planck equation, which occurs in diffusion processes. Let $0 \leqq t<\infty$, $r_{1} \leqq x \leqq r_{2}$, where $r_{1}$ is a natural boundary and $r_{2}$ an exit boundary. Let $V_{1}(t)$ and $V_{2}(t)$ result from masses at the boundaries $r_{1}$ and $r_{2}$. If $v(t, x)$ represents mass density at $x$ at time $t$, then $v(t, x)$ satisfies

$$
\begin{aligned}
v_{t} & =\left[(a(x) v)_{x}-b(x) v\right]_{x}+(\tau / \sigma) V_{2}(t) \tilde{p}(x), \\
v_{1}^{\prime}(t) & =\left(p_{1} / \sigma\right) V_{2}(t), \\
V_{2}^{\prime}(t) & =-\frac{p_{2}}{\sigma} V_{2}(t)-\lim _{x \rightarrow r_{2}}\left[(a(x) v)_{x}-b(x)\right],
\end{aligned}
$$

or when $\sigma=0$,

$$
v_{t}=\left[(a(x) v)_{x}-b(x) v\right]_{x}-p_{2}^{-1} \tau \tilde{p}(x) \lim _{x \rightarrow r_{2}}\left[(a(x) v)_{x}-b(x) v\right] .
$$

If $r_{1}$ is also an exit boundary, then there will also be a term involving a limit at $r_{1}$, or more generally there will be terms involving limits in both directions.

In the case $\sigma=0$, if $v=w(t) y(x)$, the equation in $y$ is

$$
\left[(a(x) y)^{\prime}-b(x) y\right]^{\prime}-p_{2}^{-1} \tau \tilde{p}(x)\left[(a(x) y)^{\prime}-b(x) y\right]_{x=r_{2}}=\lambda y,
$$

where $\lambda$ is the separation of variable parameter. This equation is similar to Hilb's adjoint equation.

More recently in 1964, Cole [7] discussed the vector system

$$
\begin{gathered}
L Y=Y^{\prime}+P(x) Y=\lambda Y, \\
\sum_{i=1}^{n} A_{i} Y\left(a_{i}\right)+\int_{a}^{b} K(x) Y(x) d x=0
\end{gathered}
$$

where $-\infty<a \leqq a_{1}<\cdots<a_{n} \leqq b<\infty$. He discussed the Green's function, found eigenvalues and associated eigenfunctions, and then derived an eigenfunction expansion. But most important for our purposes, he defined an adjoint operator by

$$
\begin{aligned}
& L^{+} Z=-Z^{\prime}+P^{*}(x) Z+K^{*}(x) \phi=\lambda Z, \\
& Z\left(a_{i}+\right)-Z\left(a_{i}-\right)=A_{i}^{*} \phi, \quad i=1, \ldots, n, \\
& Z\left(a_{0}-\right)=Z\left(a_{n}{ }^{+}\right)=0,
\end{aligned}
$$


where $\phi$ is a matrix parameter. Although Cole did not show it, $\phi$ can be expressed as a linear combination of the boundary values of $Z\left(Z(a), Z\left(a_{i} \pm\right), i=1, \ldots, n, Z(b)\right)$.

In 1965 the author [17] attempted to generalize the type of system so that the adjoint system would have the same form. He considered the system

$$
\begin{aligned}
& L Y=Y^{\prime}+P(x) Y+K_{2}(x) U_{2}(Y)=0, \\
& U_{1}(Y)+\int_{a}^{b} K_{1}(x) Y(x) d x=0,
\end{aligned}
$$

where $U_{1}(Y)=A Y(a)+B Y(b), U_{2}(Y)=C Y(a)+D Y(b)$ are independent boundary operators, and $K_{1}(x), K_{2}(x)$ are integrable matrices.

If $V_{1}(Z), V_{2}(Z)$ are the complementary boundary operators for $U_{1}(Y), U_{2}(Y)$, i.e.

$$
\int_{a}^{b}\left[Z^{*}\left(Y^{\prime}+P Y\right)-\left(-Z^{\prime}+P^{*} Z\right)^{*} Y\right] d x=V_{1}^{*}(Z) U_{1}(Y)+V_{2}^{*}(Z) U_{2}(Y)
$$

then the adjoint system is

$$
\begin{aligned}
& L^{+} Z=-Z^{\prime}+P^{*}(x) Z-K_{1}^{*}(x) V_{1}(Z)=0, \\
& V_{2}(Z)+\int_{a}^{b} K_{2}^{*}(x) Z(x) d x=0 .
\end{aligned}
$$

Compatibility conditions and the Green's function were discussed, but with certain technical difficulties. The virtue of this extension is that it permits classical selfadjointness to occur, which was previously impossible.

Even more recently Jones [12] has discussed this last system when $K_{2}(x)=0$, exploring the conditions under which it is selfadjoint in the extended sense of Bliss [2], [3] and Reid [22].

Finally the author [18] has recently derived the adjoint system for

$$
\begin{aligned}
& L Y=Y^{\prime}+P(x) Y+K_{2}(x) U_{2}(Y)=0, \\
& U_{1}(Y)+\int_{a}^{b} K_{1}(x) Y(x) d x=0
\end{aligned}
$$

in $L_{n}^{2}(a, b)$ even when $U_{1}(Y), U_{2}(Y)$ are defined by matrices which are not necessarily square. The adjoint system may be represented by

$$
\begin{aligned}
& L^{*} Z=-Z^{\prime}+P^{*}(x) Z-K_{1}^{*}(x) \phi \\
& -Z(b)+B^{*} \phi-D^{*} \int_{a}^{b} K_{2}^{*}(x) Z(x) d x=0 \\
& Z(a)+A^{*} \phi-C^{*} \int_{a}^{b} K_{2}(x) Z(x) d x=0
\end{aligned}
$$

Here again the parameter $\phi$ can be eliminated to yield the adjoint system previously defined. 
In the remainder of this article we shall discuss the theory of differential-boundary operators and systems. $\$ 2$ deals with the definition of these objects and the removal of interior points and integrals in the boundary conditions. $\$ 3$ exhibits solutions to homogeneous and nonhomogeneous problems and defines the Green's function for such problems. $\S 4$ derives the adjoint problem in a Hilbert space, gives two representations for it, and derives Green's formula. $\$ 5$ discusses selfadjoint problems in the sense of Lagrange and in the sense of Bliss-Reid and derives the eigenfunction expansions associated with these problems. $\$ 6$ discusses nonselfadjoint problems. It shows these problems are not regular in the sense of Birkhoff, but there exist other regularity conditions which in certain circumstances reduce to Birkhoff's conditions. The Green's function is then expanded and an eigenfunction expansion is derived. $\$ 7$ is concerned with extensions and unsolved problems.

II. Differential-boundary operators. (a) Let us consider a finite interval $[a, b]$ which is divided into $m$ subintervals $I_{1}, \ldots, I_{m}$ by $a_{1}, \ldots, a_{m-1}$. I.e., $a=a_{0}<a_{1} \ldots$ $<a_{m-1}<a_{m}=b$. Let $A_{i j}$ and $B_{i j}, i=1, \ldots, k, j=1, \ldots, m$, denote constant matrices and $K_{i}(x), i=1, \ldots, k$, denote matrices which are functions of $x$. If $Y(x)$ is a column vector, we define the boundary forms $M_{i} Y, i=1, \ldots, k$, by

$$
M_{i} Y=\sum_{j=1}^{m}\left[A_{i j} Y\left(a_{j-1}+\right)+B_{i j} Y\left(a_{j}-\right)\right]+\int_{a}^{b} K_{i}(x) Y(x) d x,
$$

$i=1, \ldots, k$, where $Y\left(a_{j^{ \pm}}\right)$denotes the limit of $Y(x)$ as $x$ approaches $a_{j}$ from above or below.

If $C_{i j}$ and $D_{i j}, i=1, \ldots, l, j=1, \ldots, m$, denote additional constant matrices, $H_{i}(x), i=1, \ldots, l$, denote additional matrix functions, and $P(x)$ is a continuous square matrix, we define the differential operator $L_{b}$ by

$$
L_{b} Y=Y^{\prime}+P(x) Y+\sum_{i=1}^{l} H_{i}(x)\left\{\sum_{j=1}^{m} C_{i j} Y\left(a_{j-1^{+}}\right)+D_{i j} Y\left(a_{j^{-}}\right)\right\} .
$$

We define the differential-boundary system $\mathscr{S}$ by

$$
\mathscr{S}: \begin{aligned}
& L_{b} Y=0, \\
& M_{i} Y=0, \quad i=1, \ldots, k .
\end{aligned}
$$

It was shown [18] that the proper adjoint system for $\mathscr{S}$ is

$$
\begin{gathered}
L_{b}^{+} Z=-Z^{\prime}+P^{*}(x) Z-\sum_{i=1}^{k} K_{i}^{*}(x) \Phi_{i}=0, \\
\mathscr{S}^{+}: \quad-Z\left(a_{j}-\right)+\sum_{i=1}^{k} B_{i j}^{*} \Phi_{i}-\sum_{i=1}^{l} D_{i j}^{*} \int_{a}^{b} H_{i}^{*}(x) Z(x) d x=0, \\
Z\left(a_{j-1}+\right)+\sum_{i=1}^{k} A_{i j}^{*} \Phi_{i}-\sum_{i=1}^{l} C_{i j}^{*} \int_{a}^{b} H_{i}^{*}(x) Z(x) d x=0, \quad j=1, \ldots, m,
\end{gathered}
$$

where $\Phi_{i}$ are appropriate parametric matrices. Hence we use it as the definition for $\mathscr{S}^{+}$. 
It is these systems and their nonhomogeneous counterparts we wish to study.

(b) It is possible to reduce $\mathscr{S}$ and $\mathscr{S}^{+}$to more manageable forms which do not involve interior points as boundary points. In each interval $I_{j}=\left[a_{j-1}, a_{j}\right]$ we denote the variable $x$ by $x_{j}$ as $j=1, \ldots, m$. We denote the vector $\mathscr{Y}$ by

$$
\mathscr{Y}=\left(\begin{array}{c}
Y\left(x_{1}\right) \\
Y\left(x_{2}\right) \\
\vdots \\
Y\left(x_{m}\right)
\end{array}\right)
$$

where the first components are evaluated in $I_{1}$, the second set in $I_{2}$, etc. If $\mathscr{P}$ is defined by

$$
\mathscr{P}=\left(\begin{array}{cccc}
P\left(x_{1}\right) & 0 & \cdots & 0 \\
0 & P\left(x_{2}\right) & \cdots & 0 \\
\vdots & \vdots & \ddots & \\
0 & 0 & & P\left(x_{m}\right)
\end{array}\right) \text {, }
$$

if $\mathscr{A}=\left(A_{i j}\right), \mathscr{B}=\left(B_{i j}\right), \mathscr{C}=\left(C_{i j}\right), \mathscr{D}=\left(D_{i j}\right)$, and if $\mathscr{K}=\left(K_{i}\left(x_{j}\right)\right), \mathscr{H}=\left(H_{j}\left(x_{i}\right)\right)$, then $\mathscr{S}$ is equivalent to

$$
\begin{gathered}
\mathscr{L}_{b} \mathscr{Y}=\mathscr{Y}^{\prime}+\mathscr{P} \mathscr{Y}+\mathscr{H}[\mathscr{C} \mathscr{Y}(A)+\mathscr{D} \mathscr{Y}(B)]=0, \\
\mathscr{A} \mathscr{Y}(A)+\mathscr{B} \mathscr{Y}(B)+\int_{A}^{B} \mathscr{K}(X) \mathscr{Y}(X) d X=0,
\end{gathered}
$$

where $A$ consists of the $m$-tuple $\left(a_{0^{+}}, a_{1}+\ldots, a_{m-1}\right), B$ consists of $\left(a_{1}-, a_{2}-\right.$, $\left.\ldots, a_{m}^{-}\right)$and $X$ is $\left(x_{1}, x_{2}, \ldots, x_{m}\right)$.

If $\mathscr{Z}$ and $\Phi$ are defined by

$$
\mathscr{Z}=\left(\begin{array}{c}
Z\left(x_{1}\right) \\
Z\left(x_{2}\right) \\
\vdots \\
Z\left(x_{m}\right)
\end{array}\right), \quad \Phi=\left(\begin{array}{c}
\Phi_{1} \\
\Phi_{2} \\
\vdots \\
\Phi_{n}
\end{array}\right),
$$

then $\mathscr{S}^{+}$is equivalent to

$$
\begin{aligned}
& \mathscr{L}_{b}^{+} \mathscr{Z}=-\mathscr{Z}^{\prime}+\mathscr{P} * \mathscr{Z}-\mathscr{K}^{*} \Phi=0 \\
& \mathscr{Z}(A)+\mathscr{A}^{*} \Phi-\mathscr{C}^{*} \int_{A}^{B} \mathscr{H}^{*}(X) \mathscr{Z}(X) d X=0, \\
& -\mathscr{Z}(B)+\mathscr{B}^{*} \Phi-\mathscr{D}^{*} \int_{A}^{B} \mathscr{H}^{*}(X) \mathscr{Z}(X) d X=0 .
\end{aligned}
$$

In this form the systems do not involve interior boundary points. If this does not suit the whim of the reader, we remark that each of the variables $x_{j}$ may be expressed in terms of a single variable $t$ which reduces the number of independent variables to 1. This was done previously by Mansfield [19] while studying interior point boundary conditions only. It introduces certain complications we prefer to avoid. 
We also remark that this notational stunt is so effective in handling a finite number of interior boundary points that henceforth we may assume there are no interior boundary points.

(c) It is now possible to evaluate the parameter occurring in the adjoint system. We assume that there are no interior boundary points, and that the boundary operators $A Y(a)+B Y(b)$ and $C Y(a)+D Y(b)$ form a row-wise complete independent set. $\mathscr{S}$ and $\mathscr{S}^{+}$thus have the form

$$
\begin{gathered}
L_{b} Y=Y^{\prime}+P(x) Y+H(x)[C Y(a)+D Y(b)]=0, \\
A Y(a)+B Y(b)+\int_{a}^{b} K(x) Y(x) d x=0 . \\
L Z_{b}^{+}=-Z^{\prime}+P^{*}(x) Z-K^{*}(x) \phi=0, \\
\mathscr{S}^{+}: \quad Z(a)+B^{*} \phi-C^{*} \int_{a}^{b} H^{*}(x) Z(x) d x=0, \\
-Z(b)+A^{*} \phi-D^{*} \int_{a}^{b} H^{*}(x) Z(x) d x=0 .
\end{gathered}
$$

It is well known [2] that Green's formula for $L Y=Y^{\prime}+P(x) Y$ and its adjoint $L^{+} Z=-Z^{\prime}+P^{*}(x) Z$ can be written as

$$
\begin{aligned}
\int_{a}^{b}\left[Z^{*}(L Y)-\left(L^{+} Z\right)^{*} Y\right] d x= & {[\tilde{A} Z(a)+\tilde{B} Z(b)]^{*}[A Y(a)+B Y(b)] } \\
& +[\tilde{C} Z(a)+\tilde{D} Z(b)]^{*}[C Y(a)+D Y(b)],
\end{aligned}
$$

where $\tilde{A}, \widetilde{B}, \tilde{C}, \tilde{D}$ are appropriate matrices which satisfy

$$
\left(\begin{array}{rr}
-\tilde{A}^{*} & -\tilde{C}^{*} \\
\widetilde{B}^{*} & \tilde{D}^{*}
\end{array}\right)\left(\begin{array}{ll}
A & B \\
C & D
\end{array}\right)=\left(\begin{array}{ll}
I & 0 \\
0 & I
\end{array}\right)
$$

and $I$ is the identity matrix of appropriate dimensions. Since these matrices are inverses, the order of multiplication may be reversed, yielding

$$
\begin{aligned}
-\tilde{A} A^{*}+\widetilde{B} B^{*} & =I, & & -\tilde{A} C^{*}+\tilde{B} D^{*}=0, \\
-\widetilde{C} A^{*}+\tilde{D} B^{*} & =0, & -\tilde{C} C^{*}+\tilde{D} D^{*} & =I .
\end{aligned}
$$

If the first adjoint boundary condition is multiplied by $-\tilde{A}$, the second by $\widetilde{B}$, and then they are added, we find that $\phi=[\tilde{A} Z(a)+\widetilde{B} Z(b)]$. If the first adjoint boundary condition is multiplied by $\widetilde{C}$, the second by $-\tilde{D}$, we find, upon adding, that

$$
\tilde{C} Z(a)+\tilde{D} Z(b)+\int_{a}^{b} H^{*}(x) Z(x) d x=0 .
$$

Therefore $\mathscr{S}^{+}$can be written as

$$
\mathscr{S}^{+}: \begin{aligned}
& L_{b}^{+} Z=-Z^{\prime}+P^{*}(x) Z-K^{*}(x)[\tilde{A} Z(a)+\tilde{B} Z(b)]=0, \\
& \tilde{C} Z(a)+\tilde{D} Z(b)+\int_{a}^{b} H^{*}(x) Z(x) d x=0 .
\end{aligned}
$$


(d) Even more remarkable, the differential-boundary systems $\mathscr{S}$ and $\mathscr{S}^{+}$may be written as ordinary differential systems with end point (no integrals) boundary conditions. Define $U$ by letting $U^{\prime}=K(x) Y, U(a)=A Y(a)$. Then

$$
U=\int_{a}^{x} K(\xi) Y(\xi) d \xi+A Y(a)
$$

and $U(b)=-B Y(b)$. Further let $S=C Y(a)+D Y(b)$. Then $\mathscr{S}$ is equivalent to

$$
\begin{gathered}
\left(\begin{array}{l}
Y \\
U \\
S
\end{array}\right)^{\prime}=\left(\begin{array}{rrr}
-P & 0 & -H \\
K & 0 & 0 \\
0 & 0 & 0
\end{array}\right)\left(\begin{array}{l}
Y \\
U \\
S
\end{array}\right) \\
\left(\begin{array}{crc}
A & -I & 0 \\
0 & 0 & 0 \\
C & 0 & -\frac{1}{2} I
\end{array}\right)\left(\begin{array}{c}
Y(a) \\
U(a) \\
S(a)
\end{array}\right)+\left(\begin{array}{ccc}
0 & 0 & 0 \\
B & I & 0 \\
D & 0 & -\frac{1}{2} I
\end{array}\right)\left(\begin{array}{c}
Y(b) \\
U(b) \\
S(b)
\end{array}\right)=0 .
\end{gathered}
$$

The adjoint system $\mathscr{S}^{+}$in this setting, as demanded by the theory of ordinary differential equations, is

$$
\begin{gathered}
\left(\begin{array}{l}
Z \\
V \\
T
\end{array}\right)=-\left(\begin{array}{ccc}
-P^{*} & K^{*} & 0 \\
0 & 0 & 0 \\
-H^{*} & 0 & 0
\end{array}\right)\left(\begin{array}{c}
Z \\
V \\
T
\end{array}\right) \\
\left(\begin{array}{ccc}
I & A^{*} & C^{*} \\
0 & 0 & D^{*} \\
0 & 0 & I
\end{array}\right)\left(\begin{array}{c}
Z(a) \\
V(a) \\
T(a)
\end{array}\right)+\left(\begin{array}{ccc}
0 & 0 & -C^{*} \\
I & -B^{*} & D^{*} \\
0 & 0 & I
\end{array}\right)\left(\begin{array}{c}
Z(b) \\
V(b) \\
T(b)
\end{array}\right)=0 .
\end{gathered}
$$

This is, in fact, equivalent to the preceding definition (2.11), (2.12). There is also an adjoint form similar to (2.15), (2.16) which comes from (2.13), (2.14). We will not need it.

We should finally note that in (2.15), (2.16) $Y$ is the dependent variable, $U$ the boundary condition variable, and $S$ is the boundary operator variable, but in (2.17), (2.18), while $Z$ is the dependent variable, the boundary condition variable is $T$, and the boundary operator variable is $V$. These last two are in reversed order.

We shall use these representations throughout the remainder of the paper.

III. Solutions. (a) It might be supposed that the existence of solutions to the equation

$$
Y^{\prime}+P(x) Y+H(x)[C Y(a)+D Y(b)]=0
$$

is guaranteed by the first part of its representation (2.8), or directly by defining $S=[C Y(a)+D Y(b)]$ and considering

$$
Y^{\prime}+P(x) Y+H(x) S=0, \quad S^{\prime}=0,
$$


which is a homogeneous system of ordinary differential equations. However neither of these techniques completely determines $S$, which is related to the boundary values of $Y$. This is seen in the need of both (2.15) and (2.16) in order to precisely define $S$.

In addition, if Picard's method of iteration is used, the feedback-like nature of $S$ is sufficient to force the successive iterations to diverge. Thus we proceed in a slightly different manner.

For the sake of preciseness, let us suppose that the sizes of the matrices $Y, P$, $H, C, D$ are $n \times 1, n \times n, n \times m, m \times n, m \times n$. We shall prove

\subsection{THEOREM. Equation (3.1) possesses $n$ linearly independent solutions.}

Proof. Let

$$
\begin{aligned}
G_{0}(x, t) & =\frac{1}{2} Y_{n}(x) Z_{n}(t), & & t<x, \\
& =-\frac{1}{2} Y_{n}(x) Z_{n}(t), & & t>x,
\end{aligned}
$$

where $Y_{n}$ is a fundamental matrix for $Y^{\prime}+P(x) Y=0, Z_{n}$ is a fundamental matrix for $-Z^{\prime}+Z P(x)=0$, and $Z_{n}(x) Y_{n}(x)=I$, the identity matrix. Then

$$
Y=Y_{n}(x) C_{1}-\int_{a}^{b} G_{0}(x, t) H(t) d t[C Y(a)+D Y(b)]
$$

for some $C_{1}$ and some $[C Y(a)+D Y(b)]$.

Evaluating $[C Y(a)+D Y(b)]$, we find

$$
\left[C Y_{n}(a)+D Y_{n}(b)\right] C_{1}=\left\{I+\int_{a}^{b}\left[C G_{0}(a, t)+D G_{0}(b, t)\right] H(t) d t\right\}[C Y(a)+D Y(b)]
$$

This is a set of $m$ linear equations, with $C_{1}$ possessing $n$ components. We wish to choose $[C Y(a)+D Y(b)]$ so that the equations will have a unique solution.

Let rank $\left[C Y_{n}(a)+D Y_{n}(b)\right]=r, r \leqq n$.

Case 1. $m>r$. The equations must be chosen so they are consistent. Having chosen $r$ linearly independent equations with arbitrary right sides (components of $\{\cdot\}[C Y(a)+D Y(b)])$, we find the remaining left sides are linear combinations of those chosen. Therefore the remaining right sides must be the same linear combination. This is possible, since, until now, the components of $[C Y(a)+D Y(b)]$ have been arbitrary. We now have $r$ equations in $n$ unknowns $\left(C_{1}\right)$ for which we can find solutions.

Subcase 1. $r=n$. In this instance we have $n$ equations in $n$ unknowns, and $C_{1}$ is uniquely determined in terms of the components of $[C Y(a)+D Y(b)]$.

Subcase 1. $r<n$. Here there exists a matrix $C_{1}$ with rank $n-r$ such that $\left[C Y_{n}(a)+D Y_{n}(b)\right] C_{1}=0$. If $Y=Y_{n}(x) C_{1}$, then we find $Y$ consists of $n-r$ independent columns, each of which is a solution.

The procedure outlined in Subcase 1, $n=r$, in this instance yields $r$ independent solutions. Since the difference between any two of them is a solution of the kind found in the first paragraph, we have found $n$ independent solutions. 
Case 2. $m=r$. Here we have the same situation which occurred in Case 1 after the initial reduction in the number of equations.

Case 3. $m<r$. This is impossible.

We can now state the following

3.2. THEOREM. Consider the system $\mathscr{S}$ which consists of

$$
\begin{aligned}
& Y^{\prime}+P(x) Y+H(x)[C Y(a)+D Y(b)]=0 \\
& A Y(a)+B Y(b)+\int_{a}^{b} K(x) Y(x) d x=0
\end{aligned}
$$

where the matrices $Y, P, C, D, H, A, B, K$ are $n \times 1, n \times n, m \times n, m \times n, n \times m, p \times n$, $p \times n, p \times n$. Let $Y_{0}$ be a fundamental set of solutions of (3.1), then $\mathscr{S}$ is compatible (possesses solutions) if and only if

$$
\operatorname{rank}\left[A Y_{0}(a)+B Y_{0}(b)+\int_{a}^{b} K(x) Y_{0}(x) d x\right]=r<n \text {. }
$$

If $r<n$, there exist $n-r$ solutions to $\mathscr{S}$.

The standard proof is applicable here.

(b) While preceding theorems show the existence of solutions to (3.1) and under what conditions $\mathscr{S}$ possesses solutions, the results are relatively useless for computational purposes. It is much more useful to use the representation (2.15) and $Y_{n}, Z_{n}$, the fundamental matrices for $Y^{\prime}+P(x) Y=0,-Z^{\prime}+Z P(x)=0$. In particular where $\mathscr{S}$ is incompatible (possesses no solutions), the nonhomogeneous boundary value problem

$$
N H: \begin{aligned}
& Y^{\prime}+P(x) Y+H(x)[C Y(a)+D Y(b)]=F(x), \\
& A Y(a)+B Y(b)+\int_{a}^{b} K(x) Y(x) d x=0
\end{aligned}
$$

is readily solved using the matrix representation.

We consider $\mathrm{NH}$ in matrix form

$$
\begin{gathered}
\left(\begin{array}{l}
Y \\
U \\
S
\end{array}\right)+\left(\begin{array}{ccc}
P & 0 & H \\
-K & 0 & 0 \\
0 & 0 & 0
\end{array}\right)\left(\begin{array}{l}
Y \\
U \\
S
\end{array}\right)=\left(\begin{array}{l}
F \\
0 \\
0
\end{array}\right), \\
\left(\begin{array}{ccc}
A & -I & 0 \\
0 & 0 & 0 \\
C & 0 & -\frac{1}{2} I
\end{array}\right)\left(\begin{array}{c}
Y(a) \\
U(a) \\
S(a)
\end{array}\right)+\left(\begin{array}{ccc}
0 & 0 & 0 \\
B & I & 0 \\
D & 0 & -\frac{1}{2} I
\end{array}\right)\left(\begin{array}{c}
Y(b) \\
U(b) \\
S(b)
\end{array}\right)=0 .
\end{gathered}
$$

It is readily seen that (3.3), (3.4) and (3.5), (3.6) are equivalent. 
To find the fundamental matrix for the homogeneous system (2.15), we solve (2.15) with initial conditions at $a$ of

$$
\left(\begin{array}{l}
I \\
0 \\
0
\end{array}\right),\left(\begin{array}{l}
0 \\
I \\
0
\end{array}\right) \text {, and }\left(\begin{array}{l}
0 \\
0 \\
I
\end{array}\right)
$$

These solutions, written consecutively in columns, form the fundamental matrix $\mathscr{Y}_{n}(x)$, which is

$$
\mathscr{Y}_{n}(x)=\left(\begin{array}{ccc}
Y_{n}(x) Z_{n}(a) & 0 & -\int_{a}^{x} Y_{n}(x) Z_{n}(\xi) H(\xi) d \xi \\
\int_{a}^{x} K(\xi) Y_{n}(\xi) d \xi Z_{n}(a) & I & -\int_{a}^{x} \int_{a}^{u} K(u) Y_{n}(u) Z_{n}(\xi) H(\xi) d \xi d u \\
0 & 0 & I
\end{array}\right)
$$

Applying the boundary condition (2.16), we have

3.3. THEOREM The system $\mathscr{S}$ is compatible if and only if the rank of

$$
\left(\begin{array}{crc}
A & -I & 0 \\
B Y_{n}(b) Z_{n}(a) & I & -B \int_{a}^{b} Y_{n}(b) Z_{n}(\xi) H(\xi) d \xi \\
+\int_{a}^{b} K(\xi) Y_{n}(\xi) d \xi Z_{n}(a) & & -\int_{a}^{b} \int_{a}^{n} K(\eta) Y_{n}(K) Z_{n}(\xi) H(\xi) d \xi d \eta \\
C+D Y_{n}(b) Z_{n}(a) & 0 & -D \int_{a}^{b} Y_{n}(b) Z_{n}(\xi) H(\xi) d \xi-I
\end{array}\right)
$$

In that case there exists a column matrix

$$
=r<3 n \text {. }
$$

$$
\left(\begin{array}{l}
C_{1} \\
C_{2} \\
C_{3}
\end{array}\right)
$$

such that when the boundary condition (2.16) is applied to

$$
\mathscr{Y}_{n}\left(\begin{array}{l}
C_{1} \\
C_{2} \\
C_{3}
\end{array}\right),
$$

the resulting matrix is a column of zeros.

3.4. Corollary. Let $H, C, D, A, B, K$ be $n \times n$ matrices. Then $\mathscr{S}$ is compatible if and only if

$$
\left|\begin{array}{crc}
A & -I & 0 \\
B Y_{n}(b) Z_{n}(a) & I & -B \int_{a}^{b} Y_{n}(b) Z_{n}(\xi) H(\xi) d \xi \\
+\int_{a}^{b} K(\xi) Y_{n}(\xi) d \xi Z_{n}(a) & & -\int_{a}^{b} \int_{a}^{n} K(\eta) Y_{n}(\eta) Z_{n}(\xi) H(\xi) d \xi d \eta \\
C+D Y_{n}(b) Z_{n}(a) & 0 & -D \int_{a}^{b} Y_{n}(b) Z_{n}(\xi) H(\xi) d \xi-I
\end{array}\right|=0 .
$$


We note that the solution to (3.1) as given by the fundamental matrix is

$$
Y_{0}=Y_{n}(x) Z_{n}(A) C_{1}-\int_{a}^{x} Y_{n}(x) Z_{n}(\xi) H(\xi) d \xi C_{3} .
$$

From the third line of (3.9) we see that

$$
\left[C+D Y_{n}(b) Z_{n}(a)\right] C_{1}=\left[I+D \int_{a}^{b} Y_{n}(b) Z_{n}(\xi) H(\xi) d \xi\right] C_{3}
$$

If $\left[C+D Y_{n}(b) Z_{n}(a)\right]^{-1}$ exists, $C_{1}$ may be eliminated to yield

$$
\begin{aligned}
Y_{0}=Y_{n}(x)\left[C Y_{n}(a)+D Y_{n}(B)\right]^{-1}\left[I-C Y_{n}(a) \int_{a}^{x} Z_{n}(\xi) H(\xi) d \xi\right. & \\
& \left.+D Y_{n}(b) \int_{x}^{b} Z_{n}(\xi) H(\xi) d \xi\right] C_{3},
\end{aligned}
$$

the solution previously derived in [17].

If the determinant (3.9) is nonzero, then standard variation of parameters solves (3.5)-(3.6). The first component solves (3.3)-(3.4).

We note in passing that $\mathscr{Y}_{n}(x)$ possesses an inverse which is

$$
\mathscr{Y}_{n}(x)^{-1}=\left(\begin{array}{ccc}
Y_{n}(a) Z_{n}(x) & 0 & \int_{a}^{x} Y_{n}(a) Z_{n}(\xi) H(\xi) d \xi \\
-\int_{a}^{x} K(\xi) Y_{n}(\xi) d \xi Z_{n}(x) & I & -\int_{a}^{x} \int_{\eta}^{x} K(\eta) Y_{n}(\eta) Z_{n}(\xi) H(\xi) d \xi d \eta \\
0 & 0 & I
\end{array}\right) .
$$

This, of course, solves the adjoint equation (2.17).

IV. The adjoint problem in Hilbert space. (a) In §II ((2.3)-(2.4), (2.11)-(2.12), (2.13)-(2.14)) we gave various equivalent definitions for the adjoint system $\mathscr{S}^{+}$. These forms formally satisfy an extended form of Green's formula. Therefore in the Hilbert space $L_{n}^{2}(a, b)$ if we define an operator $L_{b}$ by the expression (2.9) on a domain restricted by $(2.10)$, then the operator $L_{b}^{+}$defined by (2.13) with domain restricted by $(2.14)$ is a restriction of the adjoint operator $L_{b}^{*} . L_{b}^{+} \subseteq L_{b}^{*}$. Our purpose now is to show that the inclusion relationship is in fact an equality, and to derive the extended Green's formula.

We let $\mathscr{H}$ be the Hilbert space of $n \times 1$ matrices

$$
X=\left(\begin{array}{c}
x_{1}(x) \\
\vdots \\
x_{n}(x)
\end{array}\right), \quad Y=\left(\begin{array}{c}
y_{1}(x) \\
\vdots \\
y_{n}(x)
\end{array}\right)
$$

defined on the finite interval $[a, b]$, whose components are in $L^{2}(a, b)$ and whose inner product is given by

$$
(X, Y)=\sum_{i=1}^{n} \int_{a}^{b} x_{i}(x) \bar{y}_{i}(x) d x=\int_{a}^{b} Y^{*}(x) X(x) d x
$$


We let $P(x)$ be a continuous $n \times n$ matrix. We let $A, B, K(x)$ be $p \times n$ matrices, where $K(x)$ is row-wise in $\mathscr{H}$. We let $C, D$ be $m \times n$ matrices, and $H(x)$ be an $n \times m$ matrix which is column-wise in $\mathscr{H}$. We finally assume that $m+p=2 n$ and that the $2 n \times 2 n$ matrix

$$
\left(\begin{array}{ll}
A & B \\
C & D
\end{array}\right)
$$

has rank $2 n$. Thus the rows of $(A: B)$ and $(C: D)$ are all linearly independent.

The boundary form $M Y$ is defined by

$$
M Y=A Y(a)+B Y(b)+\int_{a}^{b} K(x) Y(x) d x .
$$

We now define the domain $D_{0}$ by letting $D_{0}$ be the set of all $n \times 1$ matrices $Y$ satisfying

1. $Y$ is in $\mathscr{H}$.

2. $Y$ is absolutely continuous in $[a, b]$.

3. $Y^{\prime}+P(x) Y$ is in $\mathscr{H}$.

We define the domain $D$ by letting $D$ be the set of all $n \times 1$ matrices $Y$ satisfying

1. $Y$ is in $D_{0}$.

2. $M Y=0$.

We now define the operator $L_{b}$ by

$$
L_{b} Y=Y^{\prime}+P(x) Y+H(x)[C Y(a)+D Y(b)]
$$

for all $Y$ in $D$.

4.1. THEOREM. $D$ is dense in $\mathscr{H}$.

Proof. The case where $K$ is identically 0 is well known. Thus we assume $K$ is not identically 0 . Let $\mathscr{H}_{0}$ be those matrices $Y$ such that

1. $Y$ is in $D_{0}$.

2. $Y(a)=0, Y(b)=0$.

3. $\int_{a}^{b} K(x) Y(x) d x=0$.

Then $\mathscr{H}_{0} \subset D \subset D_{0} \subset \mathscr{H}$. Let $\mathscr{K}$ be the subspace of $\mathscr{H}$ spanned by the columns of $K^{*}$. Then $\mathscr{H}_{0}$ is orthogonal to $\mathscr{K}$. Clearly $\mathscr{H}_{0}$ is dense in $\mathscr{K}^{\perp}$. Thus $D$ is dense in $\mathscr{K}^{\perp}$.

Now suppose $D$ is not dense in $\mathscr{H}$. There then exists an element $k$ in $\mathscr{K}, k \neq 0$, such that $\int_{a}^{b} k^{*}(x) Y(x) d x=0$ for all $Y$ in $D$. Hence $k=\sum_{i=1}^{p} \alpha_{i} K_{i}^{*}$ where $K_{i}^{*}$ denotes the columns of $K^{*}$, conjugate transposes of the rows of $K$. Thus if

$$
\alpha=\left(\begin{array}{l}
\alpha_{1} \\
\vdots \\
\alpha_{p}
\end{array}\right),
$$

$k=K^{*} \alpha$, and $k^{*}=\alpha^{*} K$. Thus for any $Y$ in $D, \alpha^{*} \int_{a}^{b} K(x) Y(x) d x=0$.

We see from (4.2) and the definition of $D$ that this implies $\alpha^{*}[A Y(a)+B Y(b)]=0$ for all $Y$ in $D$. Since the rows of $(A: B)$ are independent and $\alpha_{i}, i=1, \ldots, p$, are not 
all 0 , by relabelling we may assume that the terms $\alpha^{*}[A Y(a)+B Y(b)]$ and $\alpha^{*} \int_{a}^{b} K(x) Y(x) d x$ form the first row of the boundary condition (4.2). Thus $A_{1} Y(a)$ $+B_{1} Y(b)=0$ and $\int_{a}^{b} K_{1}(x) Y(x) d x=0$ for all $Y$ in $D$. Here again $\left(A_{1}: B_{1}\right)$ denotes the first row of $(A: B)$.

We now choose $U$ in $D_{0}$ such that

$$
\begin{aligned}
& A_{1} U(a)+B_{1} U(b) \neq 0 \\
& A_{i} U(a)+B_{i} U(b)+\int_{a}^{b} K_{i}(x) U(x) d x=0, \quad i=2, \ldots, p .
\end{aligned}
$$

We choose $V$ in $D_{0}$ such that $V(a)=0, V(b)=0, \int_{a}^{b}\left\|K_{1}^{*}-V\right\| d x<\delta$, where $\delta$ is to be chosen shortly, $\int_{a}^{b} K_{i} V d x=0, i=2, \ldots, p$. Since

$$
\int_{a}^{b} K_{1}(x) V(x) d x=\int_{a}^{b}\left\|K_{1}\right\|^{2} d x+\int_{a}^{b} K_{1}\left[V-K_{1}^{*}\right] d x
$$

if $\delta$ is sufficiently small $\int K_{1}(x) V(x) d x \neq 0$.

Now let $W=U+\beta V$, where

$$
\beta=-\left[A_{1} U(a)+B_{1} U(b)+\int_{a}^{b} K_{1}(x) U(x) d x\right] / \int_{a}^{b} K_{1}(x) V(x) d x .
$$

Then $W$ is in $D$, but $A_{1} W(a)+B_{1} W(b) \neq 0$, and we have a contradiction.

Since $D$ is dense in $\mathscr{H}$, the operator $L_{b}$ has a uniquely defined adjoint $L_{b}^{*}$ (see [23, p. 299]). We find it by employing a series of lemmas.

4.2. LEMMA. If $Z$ is in the domain of $L_{b}^{*}$, then $Z$ is absolutely continuous in $[a, b]$. There exists a linear functional matrix $\phi$ such that

$$
L_{b}^{*} Z=-Z^{\prime}+P^{*}(x) Z-K^{*}(x) \phi .
$$

Proof. If $Y$ is in $\mathscr{H}_{0}$, then

$$
\int_{a}^{b}\left(L_{b}^{*} Z\right)^{*} Y d x=\int_{a}^{b} Z^{*}(x)\left(Y^{\prime}+P(x) Y\right) d x .
$$

Thus

$$
\int_{a}^{b} Z^{*} Y^{\prime} d x=\int_{a}^{b}\left(L_{q}^{*} Z-P^{*}(x) Z\right)^{*} Y d x .
$$

Since $Y$ vanishes at $a$ and $b$, integration by parts on the right yields

$$
\int_{a}^{b}\left[Z+\int_{a}^{x}\left(L_{b}^{*} Z-P^{*}(t) Z\right)^{*} d t\right] Y^{\prime} d x=0
$$

We therefore have an expression of the form $\int_{a}^{b} J^{*} Y^{\prime} d x=0$ for all $Y$ in $H_{0}$. It is clear that $\int_{a}^{b} J^{*} Y d x=0$ if and only if $\int_{a}^{b}\left[\int_{a}^{x} J^{*} d t\right] Y^{\prime} d x=0$. In particular this implies that $Y^{\prime}$ is orthogonal to $J$ 's which are constant. If $Y^{\prime}$ is orthogonal to an element $J$ which is not constant and not an integral of an element in $\mathscr{K}$, then, 
assuming that $J$ has been made orthogonal to those elements as well, we find for $k$ in $\mathscr{K}$

$$
\begin{aligned}
\int_{a}^{b}\left[\int_{a}^{x} k^{*} d t\right] J d x & =-\int_{a}^{b} k^{*}\left[\int_{a}^{x} J d t\right] d x \\
& =0
\end{aligned}
$$

since $J$ being orthogonal to constants implies that $\int_{a}^{x} J d x$ vanishes at both $a$ and $b$. Thus $\int_{a}^{x} J d x$ is in $\mathscr{H}_{0}$ and $J$ is an acceptable $Y^{\prime}$. But then $J$ is orthogonal to itself and is 0 . Thus

$$
Z+\int_{a}^{x}\left(L_{b}^{*} Z-P^{*}(t) Z\right) d t=-\int_{a}^{x} K^{*}(t) d t \phi+C .
$$

Since the last three terms are differentiable a.e., $L_{b}^{*} Z=-Z^{\prime}+P^{*}(x) Z-K^{*}(x) \phi$.

4.3. LEMMA. If $Z$ is in the domain of $L_{b}^{*}$, then $Z$ satisfies

$$
\begin{array}{r}
Z(a)+A^{*} \phi-C^{*} \int_{a}^{b} H^{*}(x) Z(x) d x=0, \\
-Z(b)+B^{*} \phi-D^{*} \int_{a}^{c} H^{*}(x) Z(x) d x=0,
\end{array}
$$

or in nonparametric form,

$$
\widetilde{C} Z(a)+\tilde{D} Z(b)+\int_{a}^{b} H^{*}(x) Z(x) d x=0 .
$$

\section{Further}

$$
\phi=[\tilde{A} Z(a)+\tilde{B} Z(b)] .
$$

Proof. We compute

$$
\begin{aligned}
0= & \int_{a}^{b}\left[Z^{*}\left(L_{b} Y\right)-\left(L_{b}^{*} Z\right)^{*} Y\right] d x \\
= & Z^{*}(b) Y(b)-Z^{*}(a) Y(a)+\phi^{*} \int_{a}^{b} K(x) Y(x) d x \\
& +\left[\int_{a}^{b} H^{*}(x) Z(x) d x\right]^{*}[C Y(a)+D Y(b)] \\
= & {[\tilde{A} Z(a)+\tilde{B} Z(b)]^{*}[A Y(a)+B Y(b)] } \\
& +[\tilde{C} Z(a)+\tilde{D} Z(b)]^{*}[C Y(a)+D Y(b)] \\
& -\phi^{*}[A Y(a)+B Y(b)] \\
& +\left[\int_{a}^{b} H^{*}(x) A(x) d x\right]^{*}[C Y(a)+D Y(b)] \\
= & {[\tilde{A} Z(a)+\tilde{B} Z(b)-\phi]^{*}[A Y(a)+B Y(b)] } \\
& +\left[\tilde{C} Z(a)+\tilde{D} Z(b)+\int_{a}^{b} H^{*}(x) Z(x) d x\right]^{*}[C Y(a)+D Y(b)]
\end{aligned}
$$


Now for each nonzero row $K_{i}$, the $i$ th component of the first $Y$ boundary condition may be arbitrary. Thus the $i$ th component of $\phi$ is determined. If the $i$ th row of $K$, $K_{\mathfrak{i}}$, is 0 , then the $i$ th component of $\phi$ is unneeded and may be defined as indicated. Since the second $Y$ boundary condition is arbitrary, $Z$ satisfies (4.7).

If we use the relations concerning $A, B, C, D, \tilde{A}, \widetilde{B}, \widetilde{C}, \tilde{D}$ in $\S 2$, the conditions (4.6) follow from (4.7) and (4.8).

We now denote by $D_{0}^{*}$ those $n \times 1$ matrices $Z$ which satisfy

1. $Z$ is in $\mathscr{H}$.

2. $Z$ is absolutely continuous in $[a, b]$.

3. $-Z^{\prime}+P^{*}(x) Z$ is in $\mathscr{H}$.

We denote by $D^{*}$ those $n \times 1$ matrices $Z$ which satisfy

1. $Z$ is in $D_{0}^{*}$.

$$
\text { 2. } \tilde{C} Z(a)+\tilde{D} Z(b)+\int_{a}^{b} H^{*}(x) Z(x) d x=0 \text {. }
$$

The preceding lemmas have shown that the operator $-Z^{\prime}+P^{*}(x) Z-K^{*}(x)$ $\times[\tilde{A} Z(a)+\widetilde{B} Z(b)]$, whose domain is $D^{*}$, contains $L_{b}^{*}$. The converse of this statement is a trivial computation. We are therefore led to

4.4. ThEOREM. The domain of $L_{b}^{*}$ is $D^{*}$. For all $Z$ in $D^{*}$,

$$
L_{b}^{*} Z=-Z^{\prime}+P^{*}(x) Z-K^{*}(x)[\tilde{A} Z(a)+\widetilde{B} Z(b)]
$$

The reason for generalizing the operator (adding the boundary term) should now be clear: the adjoint operator has the same form as the original. The domains are also determined by the same kind of boundary conditions.

(b) We can now write Green's formula for these differential-boundary operators.

4.5. Theorem. Let $Y$ be in $D_{0}$ and $Z$ be in $D_{0}^{*}$. Then

$$
\begin{aligned}
& \int_{a}^{b}\left\{Z ^ { * } \left(Y^{\prime}+P(x) Y\right.\right.+H(x)[C Y(a)+D Y(b)]) \\
&\left.-\left(-Z^{\prime}+P^{*}(x) Z-K^{*}(x)[\tilde{A} Z(a)+\tilde{B} Z(b)]^{*} Y\right)\right\} d x \\
&= {[\tilde{A} Z(a)+\widetilde{B} Z(b)]^{*}\left[A Y(a)+B Y(b)+\int_{a}^{b} K(x) Y(x) d x\right] } \\
&+\left[\tilde{C} Z(a)+\tilde{D} Z(b)+\int_{a}^{b} H^{*}(x) Z(x) d x\right]^{*}[C Y(a)+D Y(b)]
\end{aligned}
$$

The proof easily follows from the comments preceding (2.13)-(2.14). This formula was derived earlier in [17] under more restrictive conditions.

4.6. Corollary. Let $Y$ be in $D$ and $Z$ be in $D^{*}$. Then

$$
\int_{a}^{b}\left[Z^{*}\left(L_{b} Y\right)-\left(L_{b}^{*} Z\right)^{*} Y\right]=0
$$


We comment finally that since the system $\mathscr{S}^{+}$generates $L_{b}^{*}$ by $L_{b}^{+}$(see 2.13 ) and generates $D^{*}$ by the boundary condition (see 2.14 ), we will no longer observe any distinction between the concepts of defined adjoint system and adjoint operator.

V. Selfadjoint problems. (a) Systems are selfadjoint in the sense of Lagrange if $L_{b}=L_{b}^{*}$. Because of the change in the sign of the derivative term, however, this is impossible without some modification. It is therefore convenient to replace $L_{b}$ and $L_{b}^{*}$ by

$$
\begin{aligned}
T_{b} Y & =(1 / i) Y^{\prime}+P(x) Y+H(x)[C Y(a)+D Y(b)], \\
T_{b}^{*} Z & =(1 / i) Z^{\prime}+P^{*}(x) Z-K^{*}(x)[\tilde{A} Z(a)+\widetilde{B} Z(b)] .
\end{aligned}
$$

$Y$ in $D$ and $Z$ in $D^{*}$ are determined by boundary conditions (4.2) and (4.9) as before, but the coefficients $\tilde{A}, \widetilde{B}, \widetilde{C}, \tilde{D}$ must be modified so that

$$
\begin{aligned}
\int_{a}^{b} & \left\{Z^{+}\left[(1 / i) Y^{\prime}+P(x) Y\right]-\left[(1 / i) Z^{\prime}+P^{*}(x) Z\right]^{*} Y\right\} d x \\
& =[\tilde{A} Z(a)+\tilde{B} Z(b)]^{*}[A Y(a)+B Y(b)]+[\tilde{C} Z(a)+\tilde{D} Z(b)]^{*}[C Y(a)+D Y(b)]
\end{aligned}
$$

Selfadjointness now occurs when $T_{b}=T_{b}^{*}$.

5.1. THEOREM. $T_{b}$ is selfadjoint if and only if

1. $P(x)=P^{*}(x)$.

2. $m=p=n$. Thus all matrices are $n \times n$ matrices.

3. $K(x)=-i\left[A C^{*}-B D^{*}\right] H^{*}(x)$.

4. $A A^{*}=B B^{*}$.

5. $H(x)\left[C C^{*}-D D^{*}\right]=0$.

We note that if $H(x)$ (or $K(x)) \equiv 0$, conditions $1-5$ reduce to the well-known results for ordinary differential systems.

Proof. Let $T_{b}$ be selfadjoint. Then 1 is trivial. 2 results from comparing the number of boundary conditions defining $D$ and $D^{*}$. To prove 3 we note that for $Y$ in $D$, both

$$
\begin{aligned}
& A Y(a)+B Y(b)=-\int_{a}^{b} K(x) Y(x) d x \\
& \tilde{C} Y(a)+\tilde{D} Y(b)=-\int_{a}^{b} H^{*}(x) Y(x) d x
\end{aligned}
$$

are satisfied. Since the rows on the left side form $n$ linearly independent boundary conditions each, there must exist a nonsingular constant matrix $E$ such that $A=E \widetilde{C}$ and $B=E \tilde{D}$. This implies that $\int_{a}^{b}\left[E H^{*}(x)-K(x)\right] Y(x) d x=0$. However, since $D$ is 
dense in $\mathscr{H}, E H^{*}(x)-K(x)=0$. Now (5.3) implies that

$$
\begin{aligned}
-\tilde{A} A^{*}+\tilde{B} B^{*} & =-i I, \\
-\tilde{A} C^{*}+\tilde{B} D^{*} & =0 \\
-\tilde{C} A^{*}+\tilde{D} B^{*} & =0 \\
-\tilde{C} C^{*}+\tilde{D} D^{*} & =-i I .
\end{aligned}
$$

Using the last equation, we find $E=-i\left[A C^{*}-B D^{*}\right]$. Thus we have established 3 and also derived

$$
\begin{aligned}
A & =-i\left[A C^{*}-B D^{*}\right] \tilde{C}, \\
B & =-i\left[A C^{*}-B D^{*}\right] \tilde{D} .
\end{aligned}
$$

From these, 4 immediately follows.

To prove 5 , we note that

$$
\left[H(x) C+K^{*}(x) \tilde{A}\right] Y(a)+\left[H(x) D+K^{*}(x) \tilde{B}\right] Y(b)=0 .
$$

Since this is an extra constraint on all $Y$ in $D$, the coefficients must vanish, and

$$
H(x) C+K^{*}(x) \tilde{A}=0, \quad H(x) D+K^{*}(x) \tilde{B}=0 .
$$

We multiply the first by $C^{*}$, the second by $D^{*}$ and subtract.

Conversely, we first note that (5.8), (5.9), and (5.6) imply (5.12), and (5.10), (5.11) and (5.6) imply (5.13). Further we note that since $\left(\begin{array}{ll}A & B \\ C & D\end{array}\right)$ is nonsingular, so is $\left(-A_{B^{*}}-{ }_{-D^{*}}\right)$. Therefore so is their product, which is

$$
\left(\begin{array}{ll}
A A^{*}-B B^{*} & A C^{*}-B D^{*} \\
C A^{*}-D B^{*} & C C^{*}-D D^{*}
\end{array}\right)
$$

Since the upper left is 0 , this is nonsingular only when $A C^{*}-B D^{*}$ is nonsingular.

Now

$$
\begin{aligned}
-K^{*}(x)[\tilde{A} Y(a)+\tilde{B} Y(b)]= & -i H(x)\left[C A^{*}-D B^{*}\right][A Y(a)+B Y(b)], \\
= & H(x)\left\{C+i\left[C C^{*}-D D^{*}\right] \tilde{C}\right\} Y(a) \\
& +H(x)\left\{D+i\left[C C^{*}-D D^{*}\right] \tilde{D}\right\} Y(b), \\
= & H(x)[C Y(a)+D Y(b)] .
\end{aligned}
$$

Thus the operators agree in form. Further

$$
\begin{aligned}
A Y(a)+B Y(b)+\int_{a}^{b} K(x) Y(x) d x & \\
= & -i\left[A C^{*}-B D^{*}\right]\left[\tilde{C} Y(a)+\tilde{D} Y(b)+\int_{a}^{b} H^{*}(x) Y(x) d x\right] .
\end{aligned}
$$

So $D$ and $D^{*}$ are the same, and $T_{b}=T_{b}^{*}$. 
At this point the situation becomes so remarkably similar to the case of selfadjoint ordinary differential systems that we only need to point to those results for proofs. Specifically, Coddington and Levinson [6, pp. 188-201] is an excellent reference. Therefore we only state the results.

5.2. TheOREM. Let $T_{b}$ be selfadjoint. Then

1. The spectrum of $T_{b}$ consists of a denumerable set of real eigenvalues with $\infty$ as their only limit point.

2. Each eigenvalue corresponds to at most $n$ eigenfunctions. Eigenfunctions corresponding to different eigenvalues are orthogonal.

3. For each complex number $\lambda$, not an eigenvalue, $\left(T_{b}-\lambda I\right)^{-1}$ exists and can be represented by a unique linear integral operator

$$
\left(T_{b}-\lambda I\right)^{-1} f(x)=\int_{a}^{b} G(\lambda, x, \xi) f(\xi) d \xi
$$

4. The Green's function $G(\lambda, x, \xi)$ satisfies

a. $G(\lambda, x, \xi)$ is continuous in $(\lambda, x, \xi)$ when $a \leqq x, \xi \leqq b, x \neq \xi$ and $\lambda$ is not an eigenvalue. For fixed $(x, \xi), G(\lambda, x, \xi)$ is meromorphic in $\lambda$.

b. $G(\lambda, x, x-0)-G(\lambda, x, x+0)=i I$.

c. As a function of $x$, for fixed $\xi$,

$$
\left(T_{b}-\lambda I\right) G(\lambda, x, \xi)=0 \text {. }
$$

d. As a function of $x$, for fixed $\xi$,

$$
M G(\lambda, x, \xi)=0 \text {. }
$$

5. The eigenfunctions of $T_{b}$ are complete in $\mathscr{H}$. If they have been arranged so that they are orthonormal (denote them by $\left.\left\{Y_{i}\right\}_{1}^{\infty}\right)$, then, when $F$ is in $D$,

$$
F=\sum_{1}^{\infty}\left(F, Y_{i}\right) Y_{i}
$$

in the sense of uniform convergence, and in the sense of convergence in the mean for all $F$ in $\mathscr{H}$.

(b) Bliss [2], [3] and Reid [22] have extended the concept of selfadjointness to systems which exhibit many of the properties of selfadjoint systems through a transformation $T(x)$. Specifically, the system

$$
\begin{aligned}
& Y^{\prime}+Q(x) Y=\lambda R(x) Y, \\
& A Y(a)+B Y(b)=0
\end{aligned}
$$

with adjoint systems

$$
\begin{aligned}
-Z^{\prime}+Q^{*}(x) Z & =\lambda R^{*}(x) Z, \\
\tilde{C} Z(a)+\tilde{D} Z(b) & =0
\end{aligned}
$$


is symmetric if there exists a nonsingular matrix $T(x)$ such that (5.20)-(5.21) is equivalent to (5.22)-(5.23) for all values of $\lambda$ under the transformation $Z=T(x) Y$. It is easy to see that this occurs if and only if

$$
\begin{aligned}
& T^{\prime}-T Q(x)-Q^{*}(x) T=0, \\
& T R(x)+R^{*}(x) T=0, \\
& A T(a)^{-1} A^{*}=B T(b)^{-1} B^{*} .
\end{aligned}
$$

Further, (5.26) is equivalent to

$$
\tilde{C}^{*} T(a) \tilde{C}=\tilde{D}^{*} T(b) \tilde{D} .
$$

The system (5.20)-(5.21) is definite if

1. It is symmetric.

2. $T^{*} R$ is hermitian and nonnegative.

3. The only solution of $Y^{\prime}+Q(x) Y=0, R(x) Y=0, A Y(a)+B Y(b)=0$ is $Y=0$. We wish to apply the results concerning definite, symmetric systems to differential-boundary systems represented in differential form by $(2.15)-(2.16)$, where $P(x)=Q(x)-\lambda R(x)$.

We note in passing that Reid [22] has shown that if $T$ exists then there exists a transformation $T_{1}$, which is skew-hermitian, under which (5.20)-(5.21) is definite and symmetric. We therefore consider skew-hermitian $T$ 's only.

5.3. THEOREM. The system

$$
\begin{gathered}
\left(\begin{array}{l}
Y \\
U \\
S
\end{array}\right)^{\prime}+\left(\begin{array}{rrr}
Q & 0 & H \\
-K & 0 & 0 \\
0 & 0 & 0
\end{array}\right)\left(\begin{array}{l}
Y \\
U \\
S
\end{array}\right)=\lambda\left(\begin{array}{lll}
R & 0 & 0 \\
0 & 0 & 0 \\
0 & 0 & 0
\end{array}\right)\left(\begin{array}{l}
Y \\
U \\
S
\end{array}\right), \\
\left(\begin{array}{ccc}
A & -I & 0 \\
0 & 0 & 0 \\
C & 0 & -\frac{1}{2} I
\end{array}\right)\left(\begin{array}{c}
Y(a) \\
U(a) \\
S(a)
\end{array}\right)+\left(\begin{array}{ccc}
0 & 0 & 0 \\
B & I & 0 \\
D & 0 & -\frac{1}{2} I
\end{array}\right)\left(\begin{array}{l}
Y(b) \\
U(b) \\
S(b)
\end{array}\right)=0
\end{gathered}
$$

is symmetric under the skew-hermitian transformation

if and only if

$$
\left(\begin{array}{ccc}
T_{11} & T_{12} & T_{13} \\
-T_{12}^{*} & T_{22} & T_{23} \\
-T_{13}^{*} & -T_{23}^{*} & T_{33}
\end{array}\right)
$$

1. (a) $T_{11}^{\prime}-T_{11} Q-Q^{*} T_{11}+T_{12} K-K^{*} T_{12}^{*}=0$.

(b) $T_{12}^{\prime}+K^{*} T_{22}-Q^{*} T_{12}=0$.

(c) $T_{13}^{\prime}-T_{11} H-Q^{*} T_{13}+K^{*} T_{23}=0$.

(d) $T_{22}^{\prime}=0$.

(e) $T_{23}^{\prime}+T_{12}^{*} H=0$.

(f) $T_{33}^{\prime}+T_{13}^{*} H-H^{*} T_{13}=0$. 
2. (a) $T_{11} R+R^{*} T_{11}=0$.

(b) $R^{*} T_{12}=0$.

(c) $R^{*} T_{13}=0$.

3. (a) $T_{11}(a)-A^{*} T_{12}^{*}(a)+T_{12}(a) A+A^{*} T_{22}(a) A=4 C^{*} T_{33}(b) C$.

(b) $T_{11}(b)+B^{*} T_{12}^{*}(b)-T_{12}(b) B+B^{*} T_{22}(b) B=4 D^{*} T_{33}(a) D$.

(c) $T_{13}(a)+A^{*} T_{23}(a)=-2 C^{*} T_{33}(a)$.

(d) $T_{13}(b)-B^{*} T_{23}(b)=-2 D^{*} T_{33}(b)$.

(e) $T_{33}(a)=T_{33}(b)$.

The proof follows from applying (5.24), (5.25) and (5.27).

5.4. THEOREM. The system (5.28)-(5.29) is definite if and only if

1. It is symmetric.

2. $T_{11}^{*} R$ is hermitian and nonnegative.

3. The only solution of

$$
\begin{aligned}
& Y^{\prime}+Q(x) Y+H(x)[C Y(a)+D Y(b)]=0 \\
& R(x) Y=0 \\
& A Y(a)+B Y(b)+\int_{a}^{b} K(x) Y(x) d x=0
\end{aligned}
$$

is $Y=0$.

Of particular interest is the situation in which $R$ is nonsingular such as in $\$ 5(a)$ where $R=i I$.

5.5. COROllary. If (5.28)-(5.29) is symmetric and $R$ is nonsingular, then (5.28)(5.29) is definite and

1. (a) $T_{11}^{\prime}-T_{11} Q-Q^{*} T_{11}=0$.

(b) $K^{*} T_{22}=0$.

(c) $-T_{11} H+K^{*} T_{23}=0$.

(d) $T_{22}^{\prime}=0$.

(e) $T_{23}^{\prime}=0$.

(f) $T_{33}^{\prime}=0$.

2. (a) $T_{11} R+R^{*} T_{11}=0$.

(b) $T_{12}=0$.

(c) $T_{13}=0$.

3. (a) $T_{11}(a)+A^{*} T_{22} A=4 C^{*} T_{33} C$.

(b) $T_{11}(b)+B^{*} T_{22} B=4 D^{*} T_{33} D$.

(c) $A^{*} T_{23}=-2 C^{*} T_{33}$.

(d) $-B^{*} T_{23}=-2 D^{*} T_{33}$.

There exist expansion theorems for symmetric, definite systems (see Bliss [2], [3]). We state the results as applied to differential-boundary systems. 
5.6. THEOREM. Let (5.28)-(5.29) be symmetric and definite. Then

1. There exists at most a denumerable set of eigenvalues, all real, with $\infty$ as their only limit point.

2. Each eigenvalue corresponds to at most $n$ eigenfunctions. Eigenfunctions corresponding to different eigenvalues ( $Y_{i}$ and $Y_{j}$ ) satisfy $\int_{a}^{b} Y_{i}^{*}(x) T_{11}^{*}(x) R(x) Y_{j}(x) d x=0$. They are orthogonal in the Hilbert space generated by the hermitian form $T_{11}^{*} R$.

3. If the eigenfunctions have been arranged so that they are orthonormal (denote them by $\left.\left\{Y_{i}\right\}\right)$, then, when $F$ satisfies

$$
\begin{gathered}
F^{\prime}+Q(x) F+H(x)[C F(a)+D F(b)]=R(x) F_{0}, \\
A F(a)+B F(b)+\int_{a}^{b} K(x) F(x) d x=0,
\end{gathered}
$$

for some continuous vector $F_{0}$, the series

$$
\mathscr{F}(x)=\sum_{i}\left[\int_{a}^{b} Y_{i}^{*}(\xi) T_{11}^{*}(\xi) R(\xi) F(\xi) d \xi\right] Y_{i}(x)
$$

converges uniformly and $R(x)[\mathscr{F}(x)-F(x)]=0$ for all $x$ in $[a, b]$.

VI. Nonselfadjoint problems. (a) The reader might expect us to turn to the classical results concerning nonselfadjoint eigenfunction expansions in this section, as was done in the previous one. This was, of course, attempted, but problems immediately resulted. In the first place, when using the representations (2.15) with $P=Q-\lambda R$, the matrix

$$
\left(\begin{array}{lll}
R & 0 & 0 \\
0 & 0 & 0 \\
0 & 0 & 0
\end{array}\right)
$$

is singular. This has never been assumed in the past. Secondly boundary conditions must be regular (see Naĭmark [20]). But as represented by (2.16), differentialboundary systems do not have regular boundary conditions. Therefore we must begin anew.

In the interest of simplifying the calculations as well as preserving our Hilbert space setting, $\mathscr{H}$, we let $P(x)=Q(x)-\lambda I$.

Further by replacing $Y$ by $\tilde{Y}$, where $Y=U \tilde{Y}$, and $U$ is a fundamental matrix for $U^{\prime}+Q(x) U=0$, we arrive at an equivalent system where $Q(x)$ is 0 .

Finally by choosing a suitable translation of $x$, we may replace the interval $[a, b]$ by $[0,1]$. We, therefore, consider the system

$$
\begin{aligned}
& L_{b} Y=Y^{\prime}+H[C Y(0)+D Y(1)]=\lambda Y, \\
& A Y(0)+B Y(1)+\int_{0}^{1} K(x) Y(x) d x=0 .
\end{aligned}
$$


(b) In order to find the eigenvalues of $L$, we note that (2.15)-(2.16) now has the form

$$
\begin{gathered}
\left(\begin{array}{l}
Y \\
U \\
S
\end{array}\right)^{\prime}=\left(\begin{array}{ccc}
0 & 0 & -H \\
K & 0 & 0 \\
0 & 0 & 0
\end{array}\right)\left(\begin{array}{l}
Y \\
U \\
S
\end{array}\right)+\lambda\left(\begin{array}{lll}
I & 0 & 0 \\
0 & 0 & 0 \\
0 & 0 & 0
\end{array}\right)\left(\begin{array}{l}
Y \\
U \\
S
\end{array}\right), \\
\left(\begin{array}{ccc}
A & -I & 0 \\
0 & 0 & 0 \\
0 & 0 & -\frac{1}{2} I
\end{array}\right)\left(\begin{array}{l}
Y(0) \\
U(0) \\
S(0)
\end{array}\right)+\left(\begin{array}{ccc}
0 & 0 & 0 \\
B & I & 0 \\
D & 0 & -\frac{1}{2} I
\end{array}\right)\left(\begin{array}{l}
Y(1) \\
U(1) \\
S(1)
\end{array}\right)=0 .
\end{gathered}
$$

Since $Y_{n}=e^{\lambda x} I$ is a fundamental matrix for $Y^{\prime}-\lambda Y=0$, the fundamental matrix for (6.3) (see 3.7) is

$$
\mathscr{Y}_{n}(x)=\left(\begin{array}{ccc}
e^{\lambda \xi} I & 0 & -\int_{0}^{x} e^{\lambda(x-\xi)} H(\xi) d \xi \\
\int_{0}^{x} e^{\lambda \xi} K(\xi) d \xi & I & -\int_{0}^{x} e^{\lambda u} K(u) \int_{0}^{u} e^{-\lambda \xi} H(\xi) d \xi d u \\
0 & 0 & I
\end{array}\right)
$$

If we let

$$
\begin{aligned}
& \mathscr{H}(x)=\int_{0}^{x} e^{-\lambda v} H(v) d v, \\
& \mathscr{K}(x)=\int_{0}^{x} e^{\lambda u} K(u) d u, \\
& \mathscr{L}(x)=\int_{0}^{x} \int_{u}^{u} e^{\lambda u} K(u) e^{-\lambda v} H(v) d v d u, \\
& \mathscr{M}(x)=\int_{0}^{x} \int_{0}^{x} e^{\lambda u} K(u) e^{-\lambda v} H(v) d v d u
\end{aligned}
$$

then

$$
\mathscr{Y}_{n}(x)=\left(\begin{array}{ccc}
e^{\lambda x} I & 0 & -e^{\lambda x} \mathscr{H}(x) \\
\mathscr{K}(x) & I & -\mathscr{L}(x) \\
0 & 0 & I
\end{array}\right)
$$

Applying the boundary condition (6.4) we see that $\lambda$ is an eigenvalue if and only if

$$
\operatorname{det}\left(\begin{array}{crc}
A & -I & 0 \\
B e^{\lambda}+\mathscr{K}(1) & I & -B e^{\lambda} \mathscr{H}(1)-\mathscr{L}(1) \\
C+D e^{\lambda} & 0 & -I-D e^{\lambda} \mathscr{H}(1)
\end{array}\right)=0 .
$$

This quickly can be reduced to yield

6.1. LEMMA. $\lambda$ is an eigenvalue of $L_{b}$ if and only if

$$
\operatorname{det}\left(\begin{array}{cc}
A+B e^{\lambda}+\mathscr{K}(1) & -B e^{\lambda} \mathscr{H}(1)-\mathscr{L}(1) \\
C+D e^{\lambda} & -I-D e^{\lambda} \mathscr{H}(1)
\end{array}\right)=0 .
$$


6.2. Lemma. Let $F$ equal the left side of (6.11). Then

1. If $\operatorname{Re}(\lambda)$ is bounded, then

$$
F=A+B e^{\lambda}+o(1)
$$

2. In any right half plane

$$
F=e^{\lambda}(-\operatorname{det} B+O(1 / \operatorname{Re}(\lambda))) \text { as } \operatorname{Re}(\lambda) \rightarrow+\infty .
$$

3. In any left half plane

$$
F=-\operatorname{det} A+O(1 / \operatorname{Re}(\lambda)) \text { as } \operatorname{Re}(\lambda) \rightarrow-\infty .
$$

Proof. These results follow rather easily by some elementary estimates and an application of the Riemann-Lebesgue Lemma.

We note that if $A$ and $B$ are nonsingular, the eigenvalues are restricted to a vertical strip. Therefore we have

\subsection{TheOREM. Let $A$ and $B$ be nonsingular. Then}

1. The eigenvalues of $L_{b}$ are restricted to a vertical strip, bounded on both the right and left.

2. The eigenvalues of $L_{b}$ are denumerable in number and become asymptotic to the zeros of $\operatorname{det}\left(A+B e^{\lambda}\right)$, which have the form

$$
\lambda_{j k}=\ln \xi_{j}+2 k \pi i,
$$

$j=1, \ldots, n, k=0, \pm 1, \pm 2, \ldots$, where $s=\xi_{j}, j=1, \ldots, n$, satisfy $\operatorname{det}(A+B s)=0$.

Proof. The last result can be found in Naĭmark's book [20].

The conditions that $A$ and $B$ be nonsingular are equivalent to the regularity conditions for ordinary differential systems. Again we refer the reader to [20]. We also note that when $A$ and $B$ are nonsingular, an argument similar to that given in Theorem 5.1 shows that $\tilde{C}$ and $\tilde{D}$ are also nonsingular.

We assume throughout the remainder of this paper that $A$ and $B$ are nonsingular.

(c) It is evident that the spectrum of $L_{b}$ consists only of eigenvalues, and these are isolated. If we consider the nonhomogeneous system similar to (6.3)-(6.4) in a somewhat more compact notation

$$
\begin{aligned}
& \mathscr{Y}^{\prime}=\mathscr{P} \mathscr{Y}+\mathscr{F}, \\
& \mathscr{A} \mathscr{Y}(0)+\mathscr{B} \mathscr{Y}(1)=0
\end{aligned}
$$

and $\lambda$ is not an eigenvalue, then $\mathscr{Y}$ is uniquely defined by the formula

$$
\mathscr{Y}(x)=\int_{0}^{1} \mathscr{G}(\lambda, x, \xi) \mathscr{F}(\xi) d \xi
$$

where

$$
\begin{aligned}
\mathscr{G}(\lambda, x, \xi) & =\mathscr{Y}_{n}(x)\left[\mathscr{A} \mathscr{Y}_{n}(0)+\mathscr{B} \mathscr{Y}_{n}(1)\right]^{-1} \mathscr{A}_{n}(0) \mathscr{Y}_{n}(\xi)^{-1}, & & \xi<x, \\
& =\mathscr{Y}_{n}(x)\left[\mathscr{A} \mathscr{Y}_{n}(0)+\mathscr{B} \mathscr{Y}_{n}(1)\right]^{-1} \mathscr{B} \mathscr{Y}_{n}(1) \mathscr{Y}_{n}(\xi)^{-1}, & & \xi>x .
\end{aligned}
$$


It is our immediate purpose to find $\mathscr{G}(\lambda, x, \xi)$ in a half plane sufficiently far to the right. Our primary interest is its upper left component, which is the Green's function for (6.1)-(6.2) and which has properties similar to those described in Theorem 5.2. First, however, we need some lemmas.

6.4. Lemma. $\operatorname{Lim}_{\operatorname{Re}(\lambda) \rightarrow+\infty} \mathscr{H}(x)=0$ uniformly for all $x$ in $[0,1]$.

\section{Proof.}

$$
\begin{aligned}
\left\|e^{\lambda x}[H(1)-H(x)]\right\| & =\left\|e^{\lambda x} \int_{x}^{1} e^{-\lambda v} H(v) d v\right\|, \\
& \leqq\left[\frac{e^{2 \operatorname{Re}(\lambda)(x-1)}-1}{-2 \operatorname{Re}(\lambda)}\right]^{1 / 2}\left[\int_{0}^{1}\|H(v)\|^{2} d v\right]^{1 / 2},
\end{aligned}
$$

which approaches 0 as $\operatorname{Re}(\lambda) \rightarrow \infty$.

6.6. LemMa. $\operatorname{Lim}_{\operatorname{Re}(\lambda) \rightarrow \infty} e^{-\lambda x} \mathscr{K}(x)=0$ uniformly for all $x$ in $[0,1]$.

Proof.

$$
\left\|\int_{0}^{x} e^{\lambda(u-x)} K(x) d x\right\| \leqq\left[\frac{1-e^{-2 \operatorname{Re}(\lambda)}}{2 \operatorname{Re}(\lambda)}\right]^{1 / 2}\left[\int_{0}^{1}\|K(u)\|^{2} d u\right]^{1 / 2},
$$

which approaches 0 as $\operatorname{Re}(\lambda) \rightarrow \infty$.

6.7. Lemma. $\operatorname{Lim}_{\operatorname{Re}(\lambda) \rightarrow \infty}[\mathscr{K}(x) \mathscr{H}(1)-\mathscr{L}(x)]=0$ uniformly for $x$ in $[0,1]$.

Proof.

$$
\begin{aligned}
\|\mathscr{K}(x) \mathscr{H}(1)-\mathscr{L}(x)\| & =\left\|\int_{0}^{x} e^{\lambda u} K(u) \int_{u}^{1} e^{-\lambda v} H(v) d v d u\right\| \\
& \leqq \int_{0}^{x} e^{\operatorname{Re}(\lambda) u}\|K(u)\|\left[\int_{u}^{1} e^{-2 \operatorname{Re}(\lambda) v} d v\right]^{1 / 2}\left[\int_{0}^{1}\|H(v)\|^{2} d v\right]^{1 / 2} d u \\
& \leqq \int_{0}^{x} e^{\operatorname{Re}(\lambda) u}\|K(u)\|\left[e^{-\operatorname{Re}(\lambda) u} /(\operatorname{Re}(\lambda))^{1 / 2}\right] d u\left[\int_{0}^{1}\|H(v)\|^{2} d u\right]^{1 / 2}, \\
& \leqq \frac{1}{\operatorname{Re}(\lambda)^{1 / 2}}\left[\int_{0}^{1}\|K(u)\|^{2} d u\right]^{1 / 2}\left[\int_{0}^{1}\|H(v)\|^{2} d v\right]^{1 / 2} .
\end{aligned}
$$

6.8. LEMMA. $\operatorname{Lim}_{\operatorname{Re}(\lambda) \rightarrow \infty} \mathscr{M}(x)=0$ uniformly for all $x$ in $[0,1]$.

The proof is similar to that of Lemma 6.7.

Now let $\Delta_{1}=\mathscr{A O}_{n}(0)+\mathscr{B} \mathscr{Y}_{n}(1)$. Then

$$
\Delta_{1}=\left(\begin{array}{crc}
A & -I & 0 \\
B e^{\lambda}+\mathscr{K}(1) & I & -B e^{\lambda} \mathscr{K}(1)-\mathscr{L}(1) \\
C+D e^{\lambda} & 0 & -D e^{\lambda} \mathscr{H}(1)-I
\end{array}\right) .
$$


Unfortunately $\Delta_{1}^{-1}$ cannot be computed directly. In addition if $\operatorname{Re}(\lambda)$ is large, it does not have any limiting value. However, if we let

$$
\Delta_{2}=\left(\begin{array}{crc}
A e^{-\lambda} & -I & A \mathscr{H}(1) \\
A e^{-\lambda}+B+e^{-\lambda} \mathscr{K}(1) & 0 & A \mathscr{H}(1)+\mathscr{M}(1) \\
C e^{-\lambda}+D & 0 & -I+C \mathscr{H}(1)
\end{array}\right)
$$

we find

$$
\Delta_{1}=\left(\begin{array}{ccc}
I & 0 & 0 \\
I & I & 0 \\
0 & 0 & I
\end{array}\right) \cdot \Delta_{2} \cdot\left(\begin{array}{ccc}
e^{-\lambda} I & 0 & \mathscr{H}(1) \\
0 & I & 0 \\
0 & 0 & I
\end{array}\right),
$$

and $\Delta_{2}$ as well as the other two matrices have limits which are invertible as $\operatorname{Re}(\lambda)$ becomes large. We can use these limits to calculate $\Delta_{1}^{-1}$ asymptotically as $\operatorname{Re}(\lambda) \rightarrow \infty$.

6.9. LEMMA.

$$
\begin{aligned}
&\left(\begin{array}{lll}
I & 0 & 0 \\
I & I & 0 \\
0 & 0 & I
\end{array}\right)^{-1}=\left(\begin{array}{rrr}
I & 0 & 0 \\
-I & I & 0 \\
0 & 0 & I
\end{array}\right) \\
&\left(\begin{array}{ccc}
e^{-\lambda} I & 0 & \mathscr{H}(1) \\
0 & I & 0 \\
0 & 0 & I
\end{array}\right)^{-1}=\left(\begin{array}{ccc}
e^{\lambda} I & 0 & -\mathscr{H}(1) e^{\lambda} \\
0 & I & 0 \\
0 & 0 & I
\end{array}\right) .
\end{aligned}
$$

As $\operatorname{Re}(\lambda)$ becomes large, $\Delta_{2}$ becomes asymptotic to

$$
\left(\begin{array}{rrr}
0 & -I & 0 \\
B & 0 & 0 \\
D & 0 & -I
\end{array}\right)
$$

which has as its inverse

$$
\left(\begin{array}{rcr}
0 & B^{-1} & 0 \\
-I & 0 & 0 \\
0 & -D B^{-1} & -I
\end{array}\right)
$$

Thus, if $\operatorname{Re}(\lambda)$ is sufficiently large,

$$
\Delta_{1}^{-1} \approx\left(\begin{array}{ccc}
e^{-\lambda} I & 0 & \mathscr{H}(1) \\
0 & I & 0 \\
0 & 0 & I
\end{array}\right)\left(\begin{array}{ccc}
0 & B^{-1} & 0 \\
-I & 0 & 0 \\
0 & D B^{-1} & -I
\end{array}\right)\left(\begin{array}{lll}
I & 0 & 0 \\
I & I & 0 \\
0 & 0 & I
\end{array}\right) .
$$

We again note that since $B^{-1}$ exists there are no eigenvalues in any half plane sufficiently far to the right. 
6.10. Lemma. If $\operatorname{Re}(\lambda)$ is sufficiently large and $\xi<x$, then

$$
\begin{aligned}
\mathscr{G}(\lambda, x, \xi) \approx & \left(\begin{array}{ccc}
e^{\lambda x} I & 0 & -e^{\lambda x} \mathscr{H}(x) \\
\mathscr{K}(x) & I & -\mathscr{L}(x) \\
0 & 0 & I
\end{array}\right)\left(\begin{array}{ccc}
e^{-\lambda} I & 0 & \mathscr{H}(1) \\
0 & I & 0 \\
0 & 0 & I
\end{array}\right)\left(\begin{array}{ccc}
0 & B^{-1} & 0 \\
-I & 0 & 0 \\
0 & D B^{-1} & -I
\end{array}\right) \\
& \cdot\left(\begin{array}{lll}
I & 0 & 0 \\
I & I & 0 \\
0 & 0 & I
\end{array}\right)\left(\begin{array}{ccc}
A & -I & 0 \\
0 & 0 & 0 \\
C & 0 & -\frac{1}{2} I
\end{array}\right)\left(\begin{array}{lll}
I & 0 & 0 \\
0 & I & 0 \\
0 & 0 & I
\end{array}\right)\left(\begin{array}{ccc}
e^{-\lambda \xi} I & 0 & \mathscr{H}(\xi) \\
-e^{-\xi} \mathscr{K}(\xi) & I & -\mathscr{M}(\xi) \\
0 & 0 & I
\end{array}\right) .
\end{aligned}
$$

The Green's function for (6.1)-(6.2), $G(\lambda, x, \xi)$, is the upper left component of this product.

$$
\begin{aligned}
G(\lambda, x, \xi) \approx & \left\{e^{\lambda(x-1)} B^{-1}+e^{\lambda x}[\mathscr{H}(1)-\mathscr{H}(x)] D B^{-1}\right\}\left\{e^{-\lambda \xi}[A+\mathscr{K}(\xi)]\right\} \\
& +\left\{-e^{\lambda x}[\mathscr{H}(1)-\mathscr{H}(x)]\right\} \cdot\left\{C e^{-\lambda \xi}\right\} .
\end{aligned}
$$

The proof is an extremely tedious computation.

6.11. Lemma. If $\operatorname{Re}(\lambda)$ is sufficiently large and $\xi>x$, then

$$
\mathscr{G}(\lambda, x, \xi) \approx\left(\begin{array}{ccc}
e^{\lambda x} I & 0 & -e^{\lambda x} \mathscr{H}(x) \\
\mathscr{K}(x) & I & -\mathscr{L}(x) \\
0 & 0 & I
\end{array}\right)\left(\begin{array}{ccc}
e^{-\lambda} I & 0 & \mathscr{H}(1) \\
0 & I & 0 \\
0 & 0 & I
\end{array}\right)\left(\begin{array}{ccc}
0 & B^{-1} & 0 \\
-I & 0 & 0 \\
0 & D B^{-1} & -I
\end{array}\right)
$$

$$
\cdot\left(\begin{array}{lll}
I & 0 & 0 \\
I & I & 0 \\
0 & 0 & I
\end{array}\right)\left(\begin{array}{ccc}
0 & 0 & 0 \\
B & I & 0 \\
D & 0 & -\frac{1}{2} I
\end{array}\right)\left(\begin{array}{ccc}
e^{\lambda} I & 0 & -e^{\lambda} \mathscr{H}(1) \\
\mathscr{K}(1) & I & -\mathscr{L}(1) \\
0 & 0 & I
\end{array}\right)\left(\begin{array}{ccc}
e^{-\lambda \xi} I & 0 & \mathscr{H}(\xi) \\
-e^{-\lambda \xi} \mathscr{K}(\xi) & I & -\mathscr{M}(\xi) \\
0 & 0 & I
\end{array}\right) .
$$

The Green's function for (6.1)-(6.2), $G(\lambda, x, \xi)$, is the upper left component of this product.

$$
\begin{aligned}
G(\lambda, x, \xi) \approx & \left\{e^{\lambda(x-1)} B^{-1}+e^{\lambda x}[\mathscr{H}(1)-\mathscr{H}(x)] D B^{-1}\right\} \cdot\left\{B e^{\lambda(1-\xi)}-e^{-\lambda \xi} \mathscr{K}(\xi)\right\} \\
& +\left\{e^{\lambda x}[\mathscr{H}(1)-\mathscr{H}(x)]\right\} \cdot\left\{D e^{\lambda(1-\xi)}\right\} .
\end{aligned}
$$

6.12. TheOREM. Let $G(\lambda, x, \xi)$ be the Green's function for (6.1)-(6.2) in a half plane sufficiently far to the right. Then

$$
\operatorname{Lim}_{\operatorname{Re}(\lambda) \rightarrow \infty} G(\lambda, x, \xi)=0
$$

uniformly for all $x, \xi$ in $[0,1]$.

Proof. This follows from Lemmas 6.5-6.11, noting that in (6.27) and (6.29), the divergent components cancel each other.

(d) Let us now consider the behavior of $G(\lambda, x, \xi)$ in a half plane sufficiently far to the left. In order to avoid a repetition of the terrible calculation of the preceding 
section we turn to a discussion of the Green's function, $G_{*}(\lambda, x, \xi)$, for the adjoint system

$$
\begin{aligned}
& L_{b}^{*} Z=-Z^{\prime}-K^{*}(x)[\tilde{A} Z(a)+\tilde{B} Z(b)]=\lambda Z, \\
& \tilde{C} Z(0)+\tilde{D} Z(1)+\int_{0}^{1} H^{*}(x) Z(x) d x=0 .
\end{aligned}
$$

Let $\lambda$ lie in a half plane sufficiently far to the left. Then $G_{*}(\lambda, x, \xi)$ is the kernel of the integral operator which generates solutions to

$$
-Z^{\prime}-K^{*}(x)[\tilde{A} Z(a)+\tilde{B} Z(b)]-\lambda Z=F
$$

which also satisfy (6.32). We have previously shown that if $\lambda$ lies sufficiently far to the left, it is in the resolvent set of $L_{b}$ and thus $L_{b}^{*}$.

Now notice that if (6.33) is multiplied by -1 , we have

$$
Z^{\prime}+K^{*}(x)[\tilde{A} Z(a)+\tilde{B} Z(b)]-(-\lambda) Z=-F,
$$

which is of the same form as the nonhomogeneous form of $(6.1)$. Further $(-\lambda)$ is in a right half plane. Thus we conclude that $\lim _{\operatorname{Re}(\lambda) \rightarrow-\infty} G_{*}(\lambda, x, \xi)=0$ uniformly for all $x, \xi$ in $[0,1]$. Since $G(\lambda, x, \xi)=-G(\lambda, \xi, x)^{*}$, and $\lambda$ lies in a left half plane when $\lambda$ does, we have

6.13. THEOREM. Let $G(\lambda, x, \xi)$ be the Green's function for (6.1)-(6.2) in a half plane sufficiently far to the left. Then

$$
\lim _{\operatorname{Re}(\lambda) \rightarrow-\infty} G(\lambda, x, \xi)=0
$$

uniformly for all $(x, \xi)$ in $[0,1]$.

We make this final comment concerning the remaining components of $\mathscr{G}(\lambda, x, \xi)$ : they, of course, do not approach 0 as $\operatorname{Re}(\lambda)$ becomes large. The reason for this is that the matrix coefficient of $\lambda$ in (6.3) is singular. Two thirds of its eigenvalues are zeros. We are indeed fortunate that $G(\lambda, x, \xi)$ is all that interests us.

(e) We now wish to expand $G(\lambda, x, \xi)$ by means of the standard technique of contour integration over a sequence of regions which ultimately enclose an arbitrarily large portion of the complex plane. In order to simplify the calculations a bit, we assume that each eigenvalue of $L_{b}$ is simple and that the eigenfunctions, $\left\{Y_{i}\right\}_{1}^{\infty}$, of $L_{b},\left\{Z_{i}\right\}_{1}^{\infty}$, of $L_{b}^{*}$, form a biorthonormal set. Multiple eigenvalues yield chains of eigenfunctions similar to those described by Naĭmark [20].

6.14. THEOREM. Let $\lambda_{0}$ be in the resolvent set of $L_{b}$. Let $\left\{\lambda_{i}\right\}_{1}^{\infty}$, be the eigenvalues of $L_{b}$ with associated eigenfunctions $\left\{Y_{i}\right\}_{1}^{\infty}$, and associated adjoint eigenfunctions $\left\{Z_{i}\right\}_{1}^{\infty}$. Assume $\int_{0}^{1} Z_{i}^{*}(x) Y_{i}(x) d x=1$ for all $i$. Then

$$
G\left(\lambda_{0}, x, \xi\right)=\sum_{i=1}^{\infty} \frac{Y_{i}(x) Z_{i}^{*}(\xi)}{\lambda_{i}-\lambda_{0}}
$$

the series converges uniformly for all $x, \xi$ in $[0,1]$. 
Proof. We evaluate $\int_{\Gamma_{n}}\left(G(\lambda, x, \xi) /\left(\lambda-\lambda_{0}\right)\right) d \lambda$, where $\Gamma_{n}$ is the following contour: First we choose $n$ sufficiently large so $\|G(\lambda, x, \xi)\|<\varepsilon / 10 \pi$ when $|\operatorname{Re}(\lambda)|>n$. This is possible in view of Theorems 6.12 and 6.13 .

Since the eigenvalues of $L_{b}$ lie in a vertical strip and are ultimately uniformly spaced apart, we can choose $\alpha_{n}$ sufficiently large so that circular arcs, centered at the origin, from $\left(-n, \alpha_{n}\right)$ to $\left(n, \alpha_{n}\right)$ and from $\left(-n,-\alpha_{n}\right)$ to $\left(\alpha,-\alpha_{n}\right)$ remain uniformly bounded away from the eigenvalues. On these arcs an application of the Riemann-Lebesgue Lemma shows that $\|G(\lambda, x, \xi)\|$ is uniformly bounded if $\alpha_{n}$ is sufficiently large. Thus if $\alpha_{n}$ is sufficiently large, on these arcs $\left\|\int G(\lambda x \xi) /\left(\lambda-\lambda_{0}\right)\right\|$ $\leqq M \Delta \theta$, where $\Delta \theta$ is the net change in the argument of $\lambda$. Clearly if $\alpha_{n}$ is sufficiently large, $\Delta \theta<\varepsilon / 4 M$.

Finally we join the ends of these arcs by semicircles centered at $(n, 0)$ and $(-n, 0)$ with radius $\alpha_{n}$ and which lie to the right of $(n, 0)$ and left of $(-n, 0)$. On these semicircles

$$
\left\|\int \frac{G(\lambda, x, \xi)}{\lambda-\lambda_{0}} d \lambda\right\|<\int \frac{\|G(\lambda, x, \xi)\|}{\left|\lambda-\lambda_{0}\right|}|d \lambda|<\varepsilon / 2
$$

if $n$ is sufficiently large.

The contour $\Gamma_{n}$ consists of these circular arcs described in a counterclockwise manner.

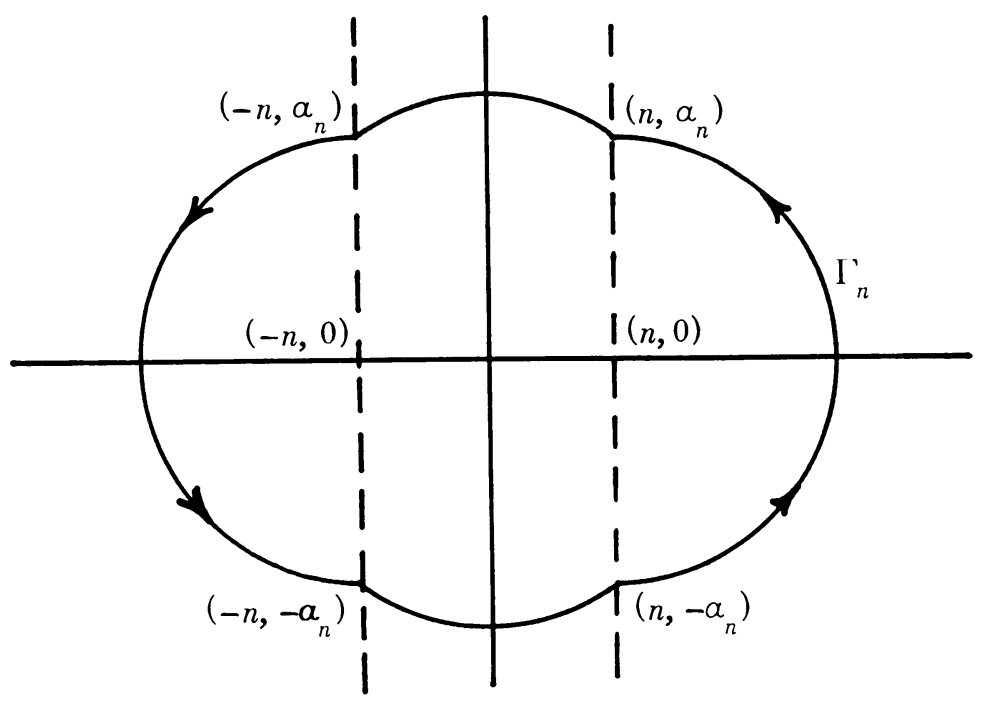

It is evident that $\left\|\int_{\Gamma_{n}}\left(G(\lambda, x, \xi) /\left(\lambda-\lambda_{0}\right)\right) d \lambda\right\|<\varepsilon$ when $n$ is sufficiently large. Thus as $n \rightarrow \infty, \int_{\Gamma_{n}}\left(G(\lambda, x, \xi) /\left(\lambda-\lambda_{0}\right)\right) d \lambda \rightarrow 0$. The result then follows by evaluating the residues of the integrand.

(f) Our final task is to express certain elements of $\mathscr{H}$ in terms of a series of eigenfunctions. We find 
6.15. THEOREM. Let the eigenfunctions of $L_{b}$ be $\left\{Y_{i}\right\}_{1}^{\infty}$ with associated adjoint eigenfunctions $\left\{Z_{i}\right\}_{1}^{\infty}$. Further assume $\int_{0}^{1} Z_{i}^{*}(x) Y_{i}(x) d x=1$. Then if $F$ is in $D$,

$$
F(x)=\sum_{i=1}^{\infty} Y_{i}(x) \int_{0}^{1} Z_{i}^{*}(\xi) F(\xi) d \xi .
$$

The series converges uniformly for all $x$ in $[0,1]$.

Proof. Let $\left(L_{b}-\lambda_{0}\right) F=F_{0}$. Then since (6.35) converges uniformly,

$$
\begin{aligned}
F(x) & =\int_{0}^{1} G\left(\lambda_{0}, x, \xi\right) F_{0}(\xi) d \xi \\
& =\sum_{i=1}^{\infty} Y_{i}(x) \int_{0}^{1} Z_{i}^{*}(\xi) F_{0}(\xi) d \xi /\left(\lambda_{n}-\lambda_{0}\right) \\
& =\sum_{i=1}^{\infty} Y_{i}(x)\left(\left(L_{b}-\lambda_{0}\right) F, Z_{i}\right) /\left(\lambda_{i}-\lambda_{0}\right), \\
& =\sum_{i=1}^{\infty} Y_{i}(x)\left(F,\left(L_{b}^{*}-\lambda_{0}\right) Z_{i}\right) /\left(\lambda_{i}-\lambda_{0}\right), \\
& =\sum_{i=1}^{\infty} Y_{i}(x)\left(F,\left(\lambda_{i}-\lambda_{0}\right) Z_{i}\right) /\left(\lambda_{i}-\lambda_{0}\right) \\
& =\sum_{i=1}^{\infty} Y_{i}(x) \int_{0}^{1} Z_{i}^{*}(\xi) F(\xi) d \xi .
\end{aligned}
$$

VII. Remarks. This essentially completes the theory of regular differentialboundary systems. Extensions to higher order systems or to those where the Hilbert space $\mathscr{H}$ is generated by another inner product are easily found by standard techniques.

There are, however, several closely related problems which are still not completely solved. First there exists only one article (Kim [13]) concerning singular differential-boundary operators as such, although there do exist some articles which nibble at the edges of the problem (see [14], [16]). The question of selfadjointness under singular conditions remains open.

Second, those problems involving boundary conditions applied at an infinite number of points are all wide open. This subject is still in its infancy. For reference we cite the articles by Whyburn [25], Bryan [4], [5], Halanay and Maro [10], Conti [8] and Stallard [24].

\section{REFERENCES}

1. Friedrich Betschler, Uber Integraldarstellungen welche aus speziellen Randwertproblemen bei gewohnlichen linearen inhomogenen Differentialgleichungen entspringen, Doctoral Dissertation, Julius-Maximilians-Universität, Wurzburg, Germany, 1912.

2. G. A. Bliss, A boundary value problem for a system of ordinary linear differential equations of the first order, Trans. Amer. Math. Soc. 28 (1926), 561-584.

3. —, Definitely self-adjoint boundary value problems, Trans. Amer. Math. Soc. 44 (1938), 413-428. 
4. R. N. Bryan, A linear differential system with general linear boundary conditions, J. Differential Equations 5 (1969), 38-48. MR 38 \#1312.

5. - A nonhomogeneous linear differential system with interface conditions, Proc. Amer. Math. Soc. 22 (1969), 270-276. MR 39 \#3078.

6. E. A. Coddington and N. Levinson, Theory of ordinary differential equations, McGrawHill, New York, 1955. MR 16, 1022.

7. R. H. Cole, General boundary conditions for an ordinary linear differential system, Trans. Amer. Math. Soc. 111 (1964), 521-550. MR 28 \#3193.

8. Roberto Conti, Recent trends in the theory of boundary value problems for ordinary differential equations, Bol. Un. Mat. Ital. (3) 22 (1967), 135-178. MR 36 \#1734.

9. W. Feller, The parabolic differential equations and the associated semi-groups of transformations, Ann. of Math. (2) 55 (1952), 468-519. MR 13, 948.

10. A. Halanay and A. Maro, $A$ boundary value problem and its adjoint, Ann. Mat. Pura Appl. (4) 79 (1968), 399-411. MR 38 \#2371.

11. E. Hilb, Über Reihentwicklungen, welche aus speciallen Randwertproblemen bei gewohnlichen linearen inhomogenen Differentialgleichungen entspringen, J. Reine Angew. Math. 140 (1911), 205-229.

12. W. R. Jones, Differential systems with integral boundary conditions, J. Differential Equations 3 (1967), 191-202. MR 34 \#6207.

13. Taeboo Kim, Investigation of a differential-boundary operator of the second order with an integral boundary condition on a semi-axis, Doctoral Dissertation, Pennsylvania State University, University Park, Pennsylvania, 1969.

14. A. M. Krall, A nonhomogeneous eigenfunction expansion, Trans. Amer. Math. Soc. 117 (1965), 352-361. MR 31 \#6020.

15. - The adjoint of a differential operator with integral boundary conditions, Proc. Amer. Math. Soc. 16 (1965), 738-742. MR 31 \#6021.

16. - Second order ordinary differential operators with general boundary conditions, Duke Math. J. 32 (1965), 617-626. MR 32 \#1397.

17. - - Nonhomogeneous differential operators, Michigan Math. J. 12 (1965), 247-255. MR 31 \#418.

18. - Differential operators and their adjoints under integral and multiple point boundary conditions, J. Differential Equations 4 (1968), 327-336. MR 37 \#6525.

19. R. Mansfield, Differential systems involving $k$-point boundary conditions, Contributions to Calculus Variations 1938-1941, Univ. of Chicago Press, Chicago, Ill., 1942, pp. 413-452. MR 4, 200.

20. M. A. NaImark, Linear differential operators, GITTL, Moscow, 1954; English transl. part I, Ungar, New York, 1967. MR 16, 702; MR 35 \#6885.

21. R. S. Phillips, Dissipative operators and hyperbolic systems of partial differential equations, Trans. Amer. Math. Soc. 90 (1959), 193-254. MR 21 \#3669.

22. W. T. Reid, A class of two-point boundary value problems, Illinois J. Math. 2 (1958), 434-453. MR 20 \#3331.

23. F. Reisz and B. Sz.-Nagy, Functional analysis, 2nd ed., Akad. Kiadó, Budapest, 1953; English transl., Ungar, New York, 1955. MR 15, 132; MR 17, 175.

24. F. W. Stallard, Functions of bounded variation as solutions of differential systems, Proc. Amer. Math. Soc. 13 (1962), 366-373. MR 25 \#2276.

25. W. M. Whyburn, Differential systems with general boundary conditions, Seminar Reports in Math., Univ. of Calif. Publ. 2 (1944), 45-61.

Pennsylvania State University,

University Park, Pennsylvania 16802 